\title{
【研究通讯】
}

\section{一类平面 $\boldsymbol{E}_{3}$ 的极限环}

$$
\begin{aligned}
& \text { 考虑平面三次系统 } \\
& \frac{\mathrm{d} x}{\mathrm{~d} t}=-y+a x+l x^{2}+m x y+n y^{2}+ \\
& p x^{3}+q x^{2} y+r x y^{2}+s y^{3}, \\
& \frac{\mathrm{d} y}{\mathrm{~d} t}=x \text {. }
\end{aligned}
$$

当 $p=q=r=s=0$ 时, (1) 式为文献 [1] 的二次微分系统的 I 类方程, 并已证明: 对于 任意的 $a, l, n$, I 类方程至多有一个极限环; 当 $l=m=n=0$ 时, (1) 式为文献 [2] 研究的平 面三次系统, 并利用二次型理论, PoincaréBendixson 定理, Levinson-Smith 定理得出一 系列结论. 本文在更大的参数范围内得到(1) 式存在极限环的充分条件.

\section{引入记号}

$$
\begin{aligned}
& \varphi(y)=y-n y^{2}-s y^{3}, \\
& F(x, y)=a+l x+m y+p x^{2}+q x y+
\end{aligned}
$$

$r y^{2}$,

$$
\Phi(y)=\int_{0}^{y} \varphi(y) \mathrm{d} y=\frac{1}{2} y^{2}-\frac{1}{3} n y^{3}-\frac{s}{4} y^{4} .
$$

则(1)式可表示为

$$
\begin{aligned}
& \frac{\mathrm{d} y}{\mathrm{~d} t}=-\varphi(y)+x F(x, y), \\
& \frac{\mathrm{d} y}{\mathrm{~d} t}=x .
\end{aligned}
$$

以下均假设 $r^{2}+p^{2}+q^{2} \neq 0(s \leqslant 0)$, 且 曲线 $F(x, y)=0$ 与曲线 $\Gamma$ 不相交. 这里

$$
\begin{gathered}
\Gamma: \frac{1}{2} x^{2}+\Phi(y)=c^{*}= \\
\begin{cases}0, & n^{2}+4 s<0, \\
\Phi(k), & n^{2}+4 s \geqslant 0,\end{cases}
\end{gathered}
$$

其中 $k=-\frac{n}{2 s}-\frac{\sqrt{n^{2}+4 s}}{2 s} \cdot \operatorname{sgn}(n)$.

取曲线族

$$
\lambda(x, y)=\frac{1}{2} x^{2}+\Phi(y)=c
$$

作地形系. 当 $n^{2}+4 s<0$ 时, (3) 式是一族包 围原点的闭曲线; 当 $n^{2}+4 s \geqslant 0$ 时, (3) 式以 $\Gamma$ 为分界线, 当 $c>\Phi(k)$ 时, $\lambda(x, y)=c$ 是 一条围绕原点且包含 $\Gamma$ 于其内部的闭曲线, 当 $c<\Phi(k)$ 时, $\lambda(x, y)=c$ 是由两个互不相 交 (可能重合)闭分枝组成, 分别位于 $\Gamma$ 内部. 借助 Poincaré-Bendixson 定理和无穷远的方

致谢本工作为国家自然科学基金(批准号: 19374039)和山东省自然科学基金(批准号: 94A0707) 资助项目.

\section{参考献}

1 Gu H P, Lou Q H, Cheung N H, et al. Experimental study of enhanced emission of the laser-ablated plume in backing gas. Appl Phys, 1994, B58: 143 148

2 Kuxuya M, Matzumoto $H$, Takechi $H$, et al. Effect of laser energy and atmosphere on the emission characteristics of laserinduced plasma. Applied Spectroscopy, 1993, 47(10): $1659 \sim 1664$

3 Sappey A D, Gamble T K. Laser-fluarescence diagnostics for condensation in laser-ablated copper plasmas. Appl Phys, 1991. B53: $353 \sim 361$

4 Befeki G, Principles of Laser Plasmas. New York: Wiley Interscience, 1976. 549 629

5 Wolf-Paul J. The plasma properties of laser-ablated $\mathrm{SiO}_{2}$. J Appl Phys, 1992, 72(4): $1280 \sim 1289$

6 Kelly R, Braren B. On the direct observation of the gas-dynamics of laser-pulse sputtering of polymers. Appl Phys, 1991. B53: $160 \sim 169$ 
【研究通讯】

\section{利用约束刻蚀剂层技术提高硅的化学刻蚀分辨率}

高分辨率刻蚀技术对于微机械及微电子 作装配的 SECM 精密定位系统, 微电极与样 器件的加工具有十分重要的意义. 虽然光刻 技术仍处于主导地位, 但近年来许多新颖的微 加工方法相继问世. 其中, 扫描电化学显微镜 (SECM) 用于表面加工的研究颇受注目 ${ }^{[1-5]}$.

SECM 刻蚀的主要原理是, 利用精密定位 系统使微电极接近样品表面, 微电极电生刻蚀 剂, 刻蚀剂扩散到样品表面发生刻蚀反应, 产 生刻蚀图样. SECM 刻蚀分辨率主要取决于 微电极的尺寸, 但刻蚀剂的横向扩散往往会对 $\mathrm{SECM}$ 的分辨率产生较大影响.

最近, 田昭武等人提出了一种可进行高分 辨率微加工的新方法一一约束刻蚀剂层技术 $(\mathrm{CELT})^{[6]}$. 其主要思路是在溶液中加入可 与电生刻蚀剂快速反应的“捕捉剂”, 使刻蚀剂 的寿命大幅度缩短, 其扩散层急剧变薄, 从而 可使刻蚀反应具有高度的距离敏感性, 刻蚀分 辨率得到极大改善.

我们利用 CELT 技术刻蚀硅表面, 与 SECM 方法相比, 刻蚀分辨率有大幅度提高.

实验采用本实验室与哈尔滨工业大学合

法 $^{[3]}$, 可得到如下结论:

定理 1 如果 $\Delta=4 p r-q^{2}>0, a r<0$, 则 (1) 式存在极限环. 且当 $a>0$ 时, 它是稳定 环; 当 $a<0$ 时, 它是不稳定环.

定理 2 如果 $\Delta=4 p r-q^{2}=0,(r+p)$. $a<0$, 则 (1) 式存在极限环. 且当 $a>0$ 时, 它 是稳定环; 当 $a>0$ 时, 它是不稳定环.

定理 3 如果 $\Delta=4 p r-q^{2}<0$, 并满足下 列条件之一：

( i ) $p a<0, p r \geqslant 0, q<0$;

(ii) $p a<0, p r \geqslant 0, q>0$ 且方程 $p u^{3}+$ $q u^{2}+r u+s=0$ 仅有一根 $u_{0}$;

(iii) $p=0, r a<0, q<0, r^{2}-8 q s \geqslant 0$, 品间距离由步进电机 $(2 \mu \mathrm{m} / \mathrm{step})$ 和压电陶瓷 (约 $60 \mathrm{~nm} / \mathrm{V}$ ) 联合控制. 三类微电极分别由 $20 \mu \mathrm{m}$ 碳纤维、60 $\mu \mathrm{m}$ 及 $100 \mu \mathrm{m}$ 铂丝制得. SECM 刻蚀电解液为 $5 \mathrm{mmol} / \mathrm{dm}^{3} \mathrm{HBr}, 0.5$ $\mathrm{mol} / \mathrm{dm}^{3} \mathrm{HF}$ 和 $0.5 \mathrm{~mol} / \mathrm{dm}^{3} \mathrm{H}_{2} \mathrm{SO}_{4}$. 进行 CELT 刻蚀实验时, 在上述溶液中加入 50 $\mathrm{mmol} / \mathrm{dm}^{3}$ 亚砷酸作为捕捉剂, 亚砷酸可与电 生 $\mathrm{Br}_{2}$ 发生快速均相反应, 达到约束刻蚀剂层 的目的. 实验用 $\mathrm{Si}$ (111) 抛光面经 $\mathrm{H}_{2} \mathrm{SO}_{4}$ 和 $\mathrm{H}_{2} \mathrm{O}_{2}$ 的 4:1 混合液洗净, 并用 $10 \% \mathrm{HF}$ 处理, 去除表面氧化膜.

Mandler 等曾利用 SECM 技术刻蚀单晶 $\mathrm{Si}(111)$ 面, 所得到的刻蚀点直径远大于所用 微电极尺寸 ${ }^{[5]}$. 我们的 SECM 刻蚀实验结果 是: 20,60 及 $100 \mu \mathrm{m}$ 直径微电极刻蚀得到的 图样直径分别约为 60,120 和 $180 \mu \mathrm{m}$. 这种 刻蚀的低分辨率主要是由刻蚀剂在微电极与 硅片之间的狭窄空间内横向扩散造成的. 当 电极反应不伴有随后均相反应时, 一般的电生 物种扩散层厚度为几十至几百微米, 这就使得

则 (1) 式存在极限环. 且当 $a>0$ 时, 它是稳 定环; 当 $a<0$ 时, 它是不稳定环.

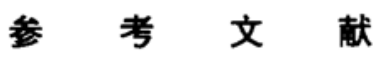

1 叶彦谦. 极限环论, 第二版. 上海: 上海科学技术出版 社, 1984. $263 \sim 284$

2 徐世龙. 关于平面 $E_{3}$ 的极限环. 科学通报, 1980,25 (10): 478

3 李 林, 应益荣, 党新益. 无穷远的意义、方法和应用. 西安: 西北大学出版社, 1996. 31 129

应益荣

(西北建筑工程学院, 西安 710061) 